\title{
Time to entry point and distal locking of intramedullary nails: a methodological phantom study comparing biplanar and uniplanar surgical imaging
}

Peter Ström, Nils P. Hailer(i) and Olof Wolf ${ }^{*}$ (1)

\begin{abstract}
Background: Intramedullary nailing is the method of choice for diaphyseal fractures of the femur and tibia and is also commonly used to treat trochanteric hip fractures. Perioperative imaging is essential for visualising adequate reduction, achieving an optimal entry point (EP) and performing distal locking (DL) of intramedullary nails. This methodological study aims to compare biplanar and uniplanar imaging in some steps of intramedullary nailing.

Methods: We used a biplanar preassembled imaging device (Biplanar ${ }^{\mathrm{TM}} 600 \mathrm{~s}$, Swemac Imaging) and a uniplanar imaging device (Ziehm Solo FD, Ziehm Imaging) to measure procedural and radiation times for antegrade and retrograde femoral and antegrade tibial nailing in fully soft flexible tissue encased legs with radiopaque sawbones (SKU:1515-7-11, Sawbones Europe, Malmö, Sweden). Four orthopaedic surgeons with different levels of experience performed all procedures in all three phantoms with both image techniques in random order, producing in total 12 EPs and nailings with DL with each imaging device. Time to EP, radiation times, time to DL for both devices and the number of swings of the uniplanar device for the two procedures were measured. Comparisons between the biplanar and uniplanar systems with a paired-samples t-test were conducted.
\end{abstract}

Results: Using the biplanar device, time to optimal EP was shorter for retrograde femoral (26 s (SD15) vs $35 \mathrm{~s}$ (SD13), $p=0.01$ ) and for antegrade tibial nailing (23 s (SD13) vs $49 \mathrm{~s}(\mathrm{SD} 24), p=0.001)$. No statistically significant differences in time to $E P$, radiation time or time to $D L$ were found for antegrade femoral nailing. A median of two swings of the uniplanar device was needed to obtain optimal EP for all procedures.

Conclusions: Biplanar imaging slightly but statistically significantly reduced time to EP for retrograde femoral and antegrade tibial nailing in this methodological study comparing biplanar and uniplanar imaging techniques. Biplanar imaging can reduce time and radiation exposure when defining the EP around the knee in intramedullary nailing procedures, but the clinical relevance of these time savings remain to be defined. For antegrade femoral nailing we found no clear benefit with biplanar imaging in the investigated steps of nailing.

Keywords: Biplanar Imaging, Uniplanar Imaging, Intramedullary Nailing, Entry Point, Distal Locking, Radiation

*Correspondence: olof.wolf@surgsci.uu.se

Department of Surgical Sciences, Orthopaedics, Uppsala University, Uppsala, Sweden

\section{Background}

Perioperative imaging is fundamental in orthopaedic trauma surgery, where uniplanar imaging is the method of choice for two-dimensional imaging. When using intramedullary devices for the treatment of femoral original author(s) and the source, provide a link to the Creative Commons licence, and indicate if changes were made. The images or other third party material in this article are included in the article's Creative Commons licence, unless indicated otherwise in a credit line to the material. If material is not included in the article's Creative Commons licence and your intended use is not permitted by statutory regulation or exceeds the permitted use, you will need to obtain permission directly from the copyright holder. To view a copy of this licence, visit http://creativecommons.org/licenses/by/4.0/. The Creative Commons Public Domain Dedication waiver (http://creativeco mmons.org/publicdomain/zero/1.0/) applies to the data made available in this article, unless otherwise stated in a credit line to the data. 
and tibial fractures uniplanar fluoroscopy is used to achieve fracture reduction, identify entry points (EP), and insert locking screws. Adequate identification of EPs is crucial to attain correct implant positioning and, as regards retrograde femoral and antegrade tibial nailing, to avoid intraarticular chondral defects $[1,2]$.

Although widely adopted, uniplanar fluoroscopy can be perceived as inefficient because of the need to rotate the $\mathrm{C}$-arm multiple times to obtain orthogonal images for all intervention steps. Simultaneous use of two C-arms to achieve good anteroposterior (AP) and lateral imaging without repositioning the fluoroscopy unit has been described [3-5]. This approach potentially reduces the need for swinging the $\mathrm{C}$-arm and may reduce the risk of surgical site contamination [4]. In Scandinavian countries, prefabricated biplanar imaging machines were introduced in the 1970s and are now widely used in orthopaedic surgery. Some other countries using biplanar imaging are Poland, China, Taiwan and Japan. A preassembled biplanar device avoids the bulky establishment of two separate $\mathrm{C}$-arms to achieve simultaneous biplanar imaging, with independent foot pedals for the respective images. Moreover, biplanar devices offer functionality and facilitate EP determination and distal locking (DL) [6].
However, it is unclear whether using a biplanar device reduces operating and radiation times compared to a conventional uniplanar device. Therefore, this methodological phantom study aims to compare time to EP and DL during ante- and retrograde intramedullary nailing of the femur and antegrade nailing of the tibia, using either preassembled biplanar imaging (Bipla$\operatorname{nar}^{\mathrm{TM}} 600$ s, Swemac Imaging, Swemac Medical Imaging Devices, Täby, Sweden) or a uniplanar C-arm (Ziehm Solo FD, Ziehm Imaging, Nuremberg, Germany) with repositioning.

\section{Methods}

This study applies a methodological framework to compare biplanar and uniplanar imaging for the times needed to conduct key steps while performing intramedullary nailing. We used intramedullary nailing of the femur (Proximal Femoral Nail Antirotation, PFNA, DePuy Synthes, Oberdorf, Switzerland) and tibia (Expert Tibial Nail, DePuy Synthes, Oberdorf, Switzerland) on fully soft tissue encased legs with radiopaque sawbones (SKU:1515-7-11, Sawbones Europe, Malmö, Sweden) (Fig. 1). Biplanar imaging was done with a preassembled device (Biplanar ${ }^{\mathrm{TM}} 600 \mathrm{~s}$, Swemac Imaging, Swemac Medical Imaging Devices, Täby, Sweden) containing two perpendicular independent image sources
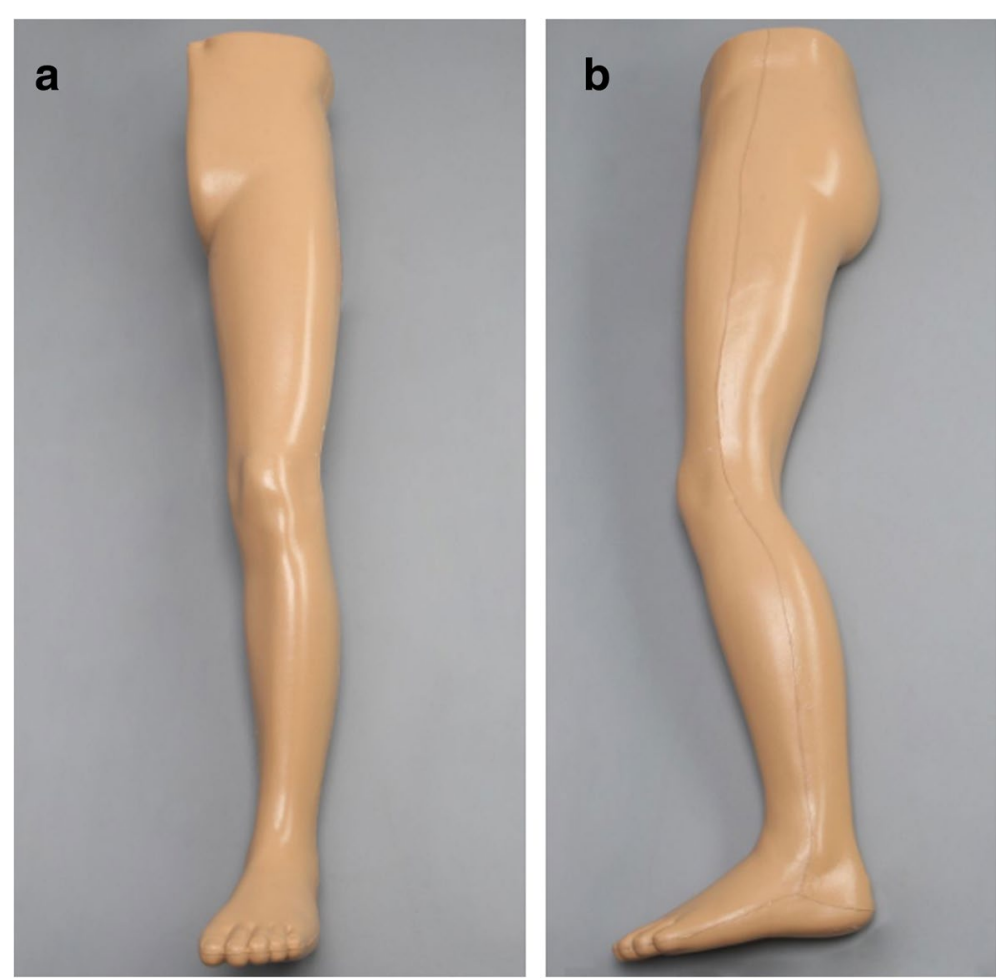

Fig. 1 Fully soft tissue encased sawbones 


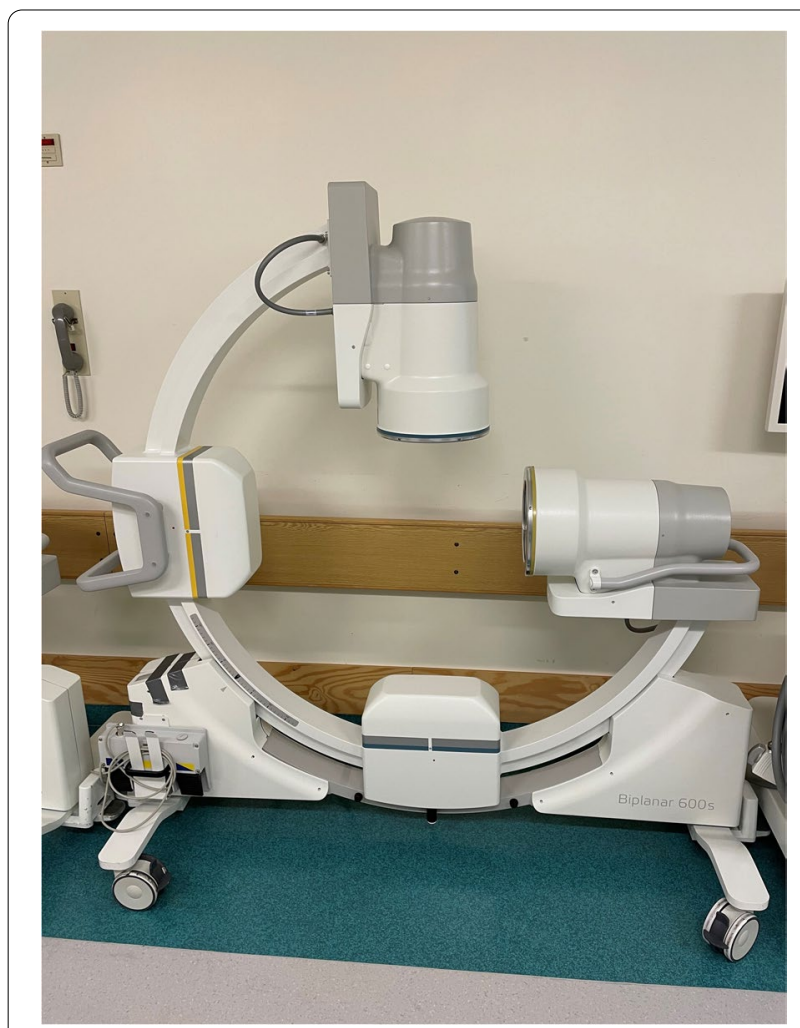

Fig. 2 Biplanar imaging (Biplanar ${ }^{\mathrm{TM}} 600$ s, Swemac Imaging) used in the study mounted in a G-stand (Fig. 2). The device allows for simultaneous AP and lateral projections displayed on separate screens (Fig. 3). For uniplanar imaging, a C-arm (Ziehm Solo FD, Ziehm Imaging, Nuremberg, Germany) was used (Fig. 4). This device is slightly smaller but requires repositioning or swinging the $\mathrm{C}$-arm to obtain AP and lateral views.

The encased legs were securely fastened and positioned on a radiolucent fracture table (Fig. 5). Three orthopaedic trauma surgeons with varying experience (1, 10 and $>15$ years post-training) and one orthopaedic trainee performed the procedures. Each surgeon executed each of three procedures: Antegrade insertion of the intramedullary femoral nail including DL, identification of EP for retrograde femoral nail and antegrade insertion of the tibial nail including DL. Each of the four surgeons performed each procedure three times on different sawbones with uniplanar imaging and three times with biplanar imaging, producing 12 sets of procedures. The four surgeons conducted the procedures in random order and times (in seconds) for procedures and radiation time were recorded. The fully soft tissue encased leg with radiopaque sawbones was dressed in surgical drapes. We measured time from skin incision to positioning of a guidewire at the tip of the greater trochanter as the optimal EP (aligned with the intramedullary femoral canal) for the PFNA, time elapsed from skin incision to bicortical drilling through the nail in preparation for one DL screw (static)

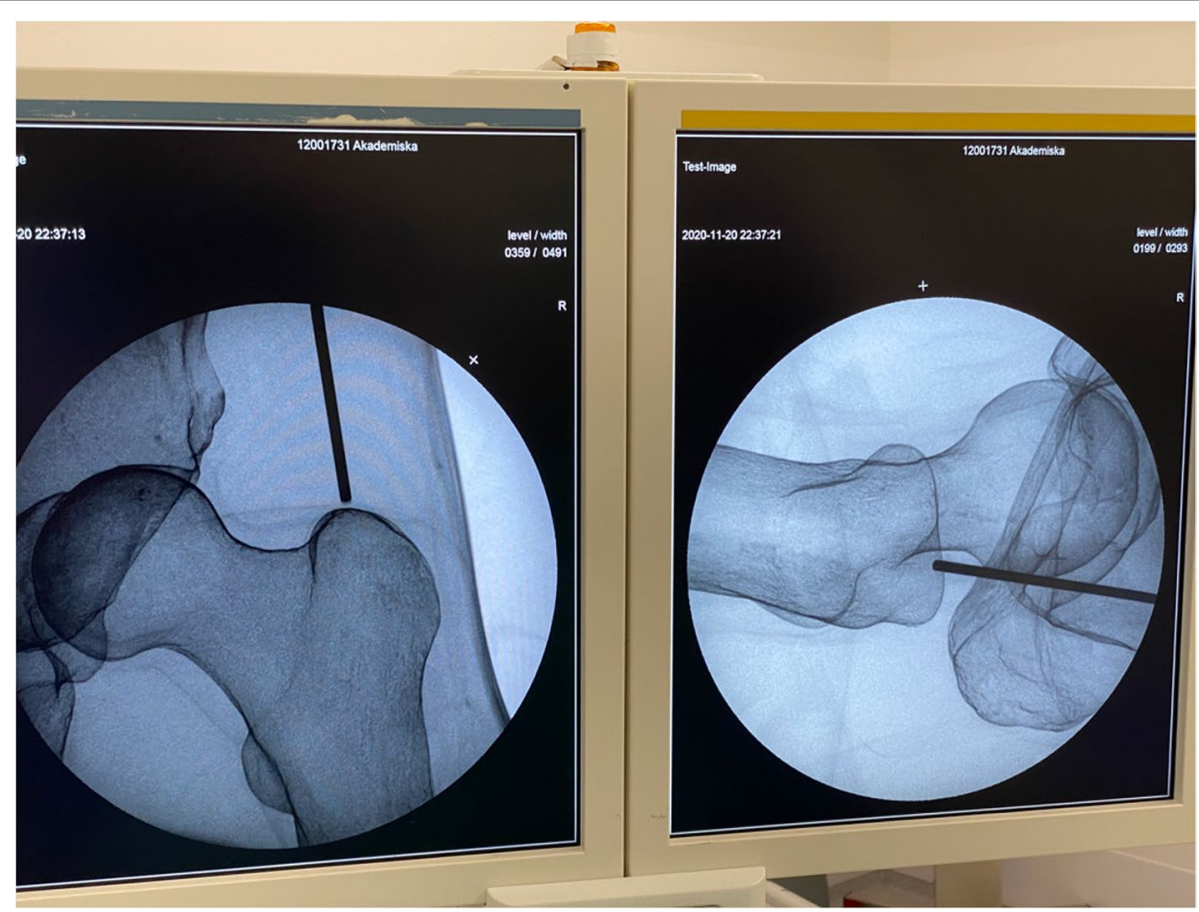

Fig. 3 Hip entry point under biplanar imaging 


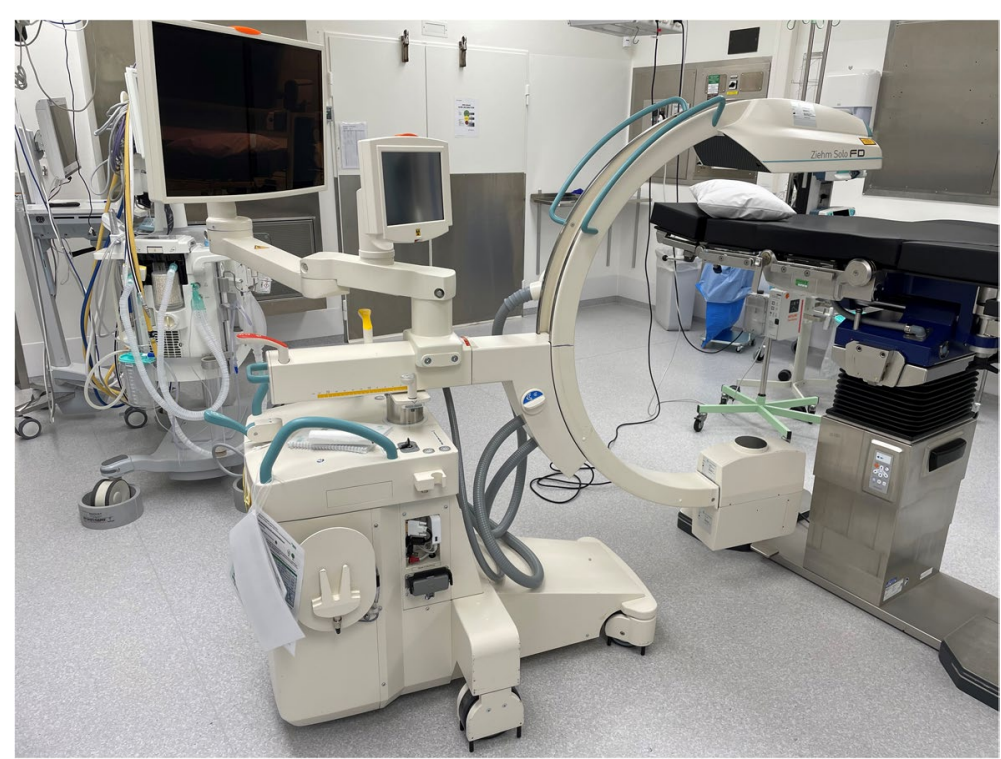

Fig. 4 Uniplanar imaging (Ziehm Solo FD, Ziehm Imaging) used in the study

through a long PFNA femoral nail, time from skin incision to guidewire positioning at the optimal EP (aligned with the intramedullary femoral canal and in extension with Blumensaat's line) for the retrograde femoral nail, time from skin incision to guidewire positioning at the

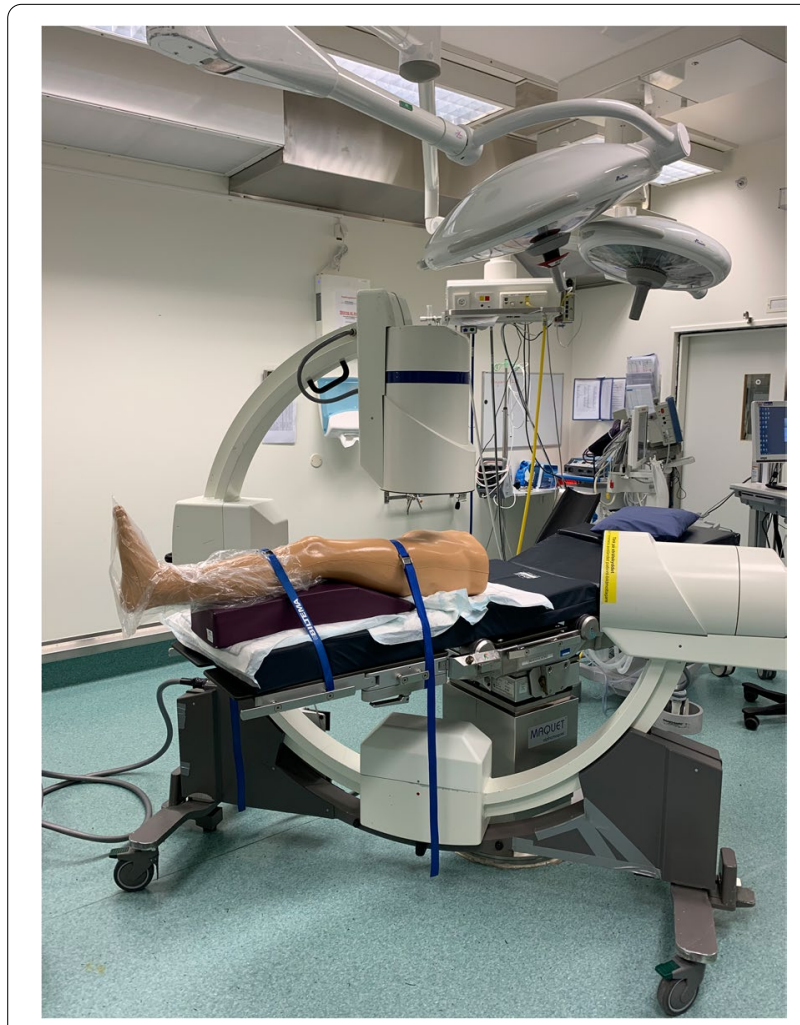

Fig. 5 Securely positioned sawbone with biplanar imaging device optimal EP (in the middle of the intercondylar notch, aligned with the axis of the intramedullary tibial canal and on the anterior edge of the proximal tibia) for the Expert Tibial Nail using a suprapatellar approach and time elapsed from skin incision to bicortical drilling through the nail in preparation for one DL screw (static). We used three sawbones and performed the distal drilling at different levels by adjusting the nail position to achieve an untouched area. DL was performed with the uniplanar imaging in the lateral position and time measurement was initiated once an optimal image had been obtained. The image intensifiers were controlled by foot control by the orthopaedic surgeon. Repositioning of uniplanar imaging was done by an assistant theatre nurse. The number of performed swings of the uniplanar imaging and the number of missed drillings (anterior or posterior to nail) for DL were noted. Accurate positioning was determined by fellow orthopaedic surgeons.

\section{Statistical analysis}

Calculation times to identify EP, bicortical drilling for the DL screw and radiation times are reported in seconds using means and standard deviations (SD) and the number of swings and missed drillings as medians and range. Differences between biplanar and uniplanar imaging were compared using the paired-samples t-test for each. Individual values for time to EP and time to DL were plotted in grouped scatter plots. All statistical calculations were made with SPSS version 27 and $P<0.05$ was considered statistically significant. 


\section{Results}

The comparison for all procedures is presented in Table 1. All 12 individual times to EP and DL for each procedure are visualised in Fig. 6.

\section{Antegrade nailing of the femur}

No statistically significant difference was found in time to EP between biplanar and uniplanar imaging. Mean time to EP was $46 \mathrm{~s}$ (SD35) for biplanar and $62 \mathrm{~s}$ (SD39) for the uniplanar device. There was also no statistically significant difference in radiation times. A median of two swings of the $\mathrm{C}$-arm was required to achieve an optimal EP into the proximal femur. Time to DL did not differ between biand uniplanar imaging, $59 \mathrm{~s}$ (SD42) versus $47 \mathrm{~s}$ (SD16). Missing the DL hole was rare for both devices.

\section{Retrograde nailing of the femur}

A statistically significantly shorter time to EP was observed with biplanar imaging (26 s (SD15)) compared to uniplanar imaging (35 s (SD13)). Radiation times did not differ between the two imaging modes. A median of two swings was used to achieve optimal EP for retrograde femoral nailing.

\section{Antegrade nailing of the tibia}

Time to EP was statistically significantly shorter for biplanar imaging, $23 \mathrm{~s}$ (SD13) vs $49 \mathrm{~s}$ (SD24). Also, radiation time for finding the optimal EP was statistically significantly shorter with biplanar imaging, $8 \mathrm{~s}$ (SD3) vs $14 \mathrm{~s}$ (SD6). A median of two swings of the uniplanar device was required to achieve an optimal EP into the proximal tibia. Time to DL for the distal tibia and radiation time for DL did not differ significantly between bi- and uniplanar imaging. No relevant difference between imaging modes in radiation times was detected. A median of one missed DL drilling was observed for biplanar imaging compared to zero missed for uniplanar imaging.

\section{Subsequent procedures and surgeon experience}

In this methodological setting times were generally fast for all 4 surgeons. We did not experience a clear trend towards faster times regarding subsequent attempts for any of the surgeons. Each surgeon had some trouble at some of the procedures, so times went in both directions without any clear trend. More missed DL screws were noted in later attempts as surgeons tried to do it faster. The two more experienced surgeons were generally faster than the more inexperienced for all procedures (see individual values, Fig. 6).

\section{Discussion}

\section{Principal findings}

This study reports the timing of different steps of an intramedullary nailing procedure of the femur or tibia.

Table 1 Mean time to entrypoint (EP) in seconds (SD; range), mean radiation time in seconds (SD; range), median number of swings of C-arm (range), and median number of missing attempts for distal locking (DL) (range). $P$ value $<0.05$ considered significant

\begin{tabular}{|c|c|c|c|}
\hline & Biplanar & C-arm & $P$ value \\
\hline \multicolumn{4}{|l|}{ Antegrade Femur } \\
\hline Time EP & $46(35 ; 15-121)$ & $62(39 ; 26-154)$ & 0.16 \\
\hline Radiation EP & $11(6 ; 7-23)$ & $14(7 ; 4-25)$ & 0.34 \\
\hline Swings EP & na & $2(1-6)$ & \\
\hline Time DL & $59(42 ; 22-144)$ & $47(16 ; 27-73)$ & 0.35 \\
\hline Radiation DL & $18(12 ; 7-44)$ & $15(5 ; 8-23)$ & 0.49 \\
\hline Swings DL & na & lateral position & \\
\hline Missing hole DL & $0(0-2)$ & $0(0-1)$ & \\
\hline \multicolumn{4}{|l|}{ Retrograde Femur } \\
\hline Time EP & $26(15 ; 8-54)$ & $35(13 ; 18-67)$ & 0.01 \\
\hline Radiation EP & $8(5 ; 2-16)$ & $10(5 ; 5-23)$ & 0.30 \\
\hline Swings EP & na & $2(2-3)$ & \\
\hline \multicolumn{4}{|l|}{ Antegrade Tibia } \\
\hline Time EP & $23(13 ; 10-52)$ & $49(24 ; 24-91)$ & 0.001 \\
\hline Radiation EP & $8(3 ; 4-13)$ & $14(6 ; 7-29)$ & 0.007 \\
\hline Swings EP & na & $2(2-6)$ & \\
\hline Time DL & $89(58 ; 23-183)$ & $78(62 ; 29-230)$ & 0.61 \\
\hline Radiation DL & $26(15 ; 7-55)$ & $24(16 ; 9-56)$ & 0.76 \\
\hline Swings DL & na & lateral position & \\
\hline Missing hole DL & $1(0-2)$ & $0(0-2)$ & \\
\hline
\end{tabular}




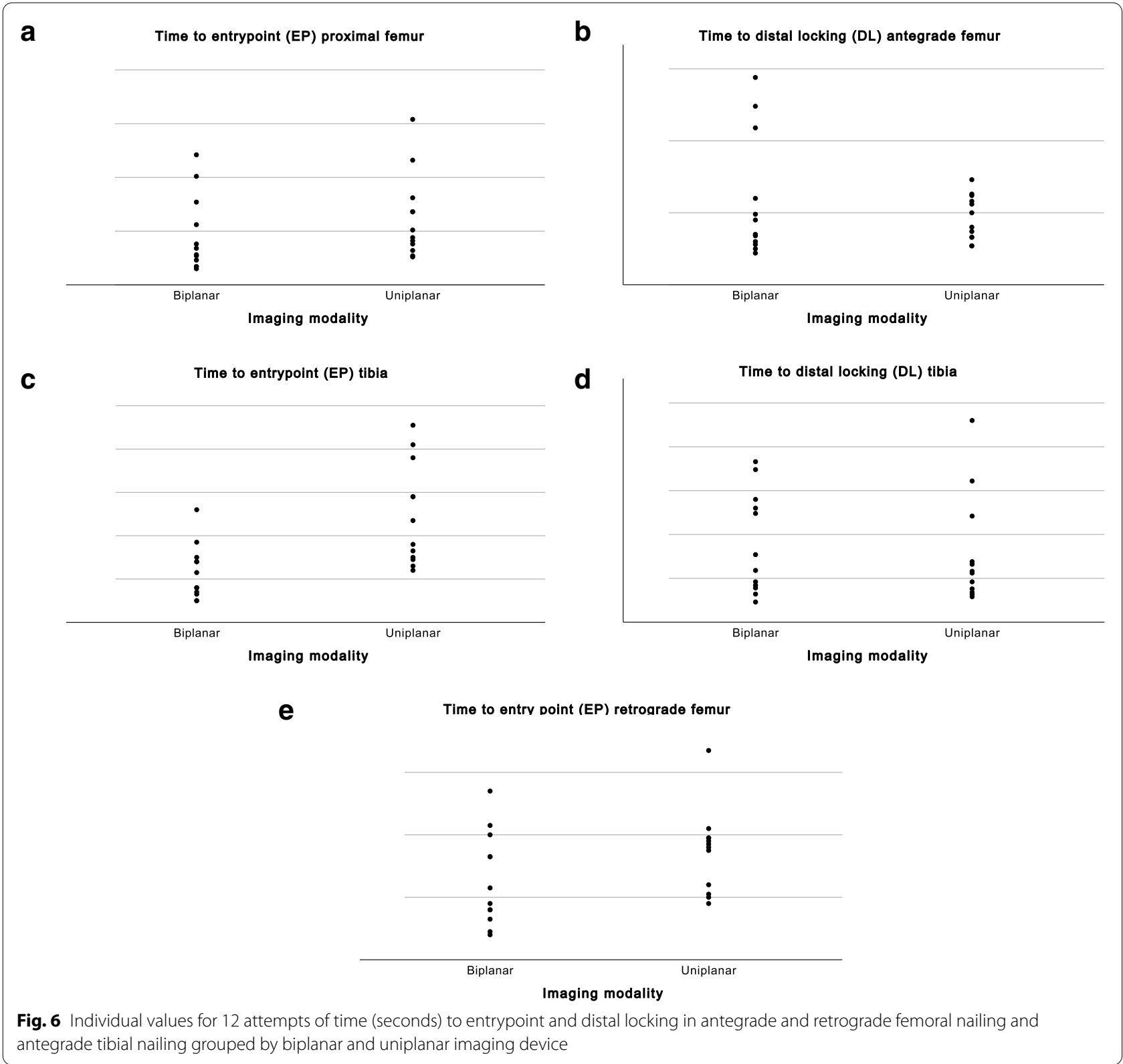

The main finding of this study is that the use of a biplanar device slightly reduced time to identify EP for tibial nailing and retrograde femoral nailing. A reduction in radiation time was found only in time to EP for the tibia but not for the femur. There was a tendency towards shorter times for DL for both antegrade femur and tibia with uniplanar imaging, although not statistically significantly so, when compared to biplanar imaging.

\section{Agreement and disagreement with the literature}

The choice of correct EPs is critical in the stabilisation of femoral or tibial fractures with intramedullary nails.
Indeed, failure to identify the right EP can cause fracture malalignment and iatrogenic fracture, such as has been described for lateral femoral nails [1]. Not all EPs are equal, as was shown when comparing fluoroscopy times required for the medial posterior and the lateral anterior trochanteric EP when using the PFNA device [7]. In antegrade femoral nailing trochanteric overhang can obstruct the approach to the piriformis fossa for nail insertion in about $25 \%$ of the patients [8]. Additionally, chondral defects in the knee that may be more frequent when using suprapatellar than parapatellar approaches can be avoided using the correct anatomical location of EPs [2]. 
Although using a uniplanar device, commonly called a $\mathrm{C}$-arm, and manual nail insertion and reaming during intramedullary nailing are clinically the most common method, alternative techniques (e.g., biplanar and robotic devices) have been explored [4, 9]. A combination of fluoroscopy and navigation has been described, but nonorthogonal imaging reduced the accuracy of EP identification, even in the presence of navigation [10].

\section{Strengths and weaknesses in relation to other studies}

We sought to investigate whether a biplanar device can reduce the time required to identify adequate EPs and the time for DL during intramedullary nailing of the femur or tibia.

We have not taken preoperative positioning and draping of real patients into consideration in this methodological study. In closed reduction in the fracture table it often takes several swings of the $\mathrm{C}$-arm to confirm reduction. When draping the $\mathrm{C}$-arm, it must swing freely so as not to contaminate the operating field or get stuck and frustrate the surgeon. Biplanar imaging is preassembled and both the reduction manoeuvre and the operating procedure can be performed without repositioning. For DL, the image sources are advanced distally and a fast and reliable technique for DL using biplanar imaging has been widely adopted [6]. Both AP and lateral image sources are draped before the procedure. There is even room for the surgeon to introduce long femoral nails between the patient and the lateral image source.

Our clinical experience is that nail procedures are easier and faster with biplanar imaging, although no objective data supports this, and, to our knowledge, there are no comparative studies to confirm this view. The simultaneous use of two $\mathrm{C}$-arms reduced the number of attempts to achieve optimal pin position in percutaneous pinning of proximal humerus fractures while reducing excess exposures and potential surgical field contamination [11]. In the repair of supracondylar humerus fractures there was no difference in fluoroscopy time or radiation dose for biplanar or uniplanar imaging using one or two $\mathrm{C}$-arms [12]. There was no significant difference in surgery times for slipped capital femoral epiphysis, but the screw positioning was better in the biplanar modus using two C-arms [3]. Both these studies are clinical, comparing surgery times and radiation doses for full procedures. Our methodological study using soft-tissue ensheathed saw bone phantoms examined steps of a full procedure using a preassembled biplanar imaging device compared to a $\mathrm{C}$-arm. When using biplanar imaging, surgeons with different levels of expertise were slightly quicker in locating the EP in the tibia and for retrograde femoral nailing, but differences were small. We found no difference in DL time; however, the time began with the uniplanar device arranged in a perfect lateral position. Such a configuration will introduce swinging of the uniplanar device with sterile drapes that can hinder free movement in the clinical setting. It is also time-consuming to find the correct true lateral projection in order to safely perform DL. Unfortunately, we did not time this set up which would have resulted in longer times for DL and thus be advantageous for the biplanar device in a direct comparison. The use of biplanar imaging for intramedullary nailing of tibia and femur has been recommended to overcome the drawbacks of uniplanar imaging, i.e. time consumption and risks of contamination [4].

The inevitable swinging of the uniplanar $\mathrm{C}$-arm confers an increased risk of surgical field contamination $[4,11]$. Contamination of the surgical field was linearly increased with increased cycles/swings of the C-arm with all areas contaminated after 15 cycles in a methodological study using fluorescent powder contaminant [13]. The area closest to the $\mathrm{C}$-arm had the most pronounced contamination. Contamination of $\mathrm{C}$-arm drapes are common, they are correlated to swinging of the $\mathrm{C}$-arm and occur early during procedures [14]. The surgeon-operated mini C-arm showed contamination of the drapes in $70 \%$ of the cases [15]. Newer draping techniques of the C-arm compared to older seem to decrease contamination [16, 17]. A future comparative study between uniplanar and biplanar imaging regarding surgical field contamination might be warranted.

Our study's methodological arrangement does not consider the full clinical reality of bleeding and drapes that move while repositioning, becoming immovable when trying to swing back. On the other hand, we found that both techniques work equally reliable in the hands of experienced as well as inexperienced orthopaedic surgeons. DL with biplanar imaging, using the technique described by Granhed in 1998 [6], is fast but requires some practising before the technique is fully understood and works in the surgeon's hands. The wide range of procedural times for DL in the distal femur nicely illustrates this fact.

The total time for the procedure is not fully accounted for by our methodological study measuring procedural steps, given that DL was measured after the $\mathrm{C}$-arm was tilted to the desired lateral position for both the distal femur and the distal tibia. With the biplanar device, one slides it down to the right location and then both AP and lateral images are displayed without much further adjustment. Based on our clinical experience with biplanar imaging, performing two or three DL screws with drilling and inserting the screws is much faster. In this methodological setting we measured the time for only one DL screw for each position.

Biplanar imaging is sometimes criticised for obstructing the surgeon's range of action in accessing the operating field. The clinical experience is that when nailing the 
femur and tibia, it is rarely necessary to move the biplanar image sources other than along the extremity. One can preoperatively widen the distance of the lateral image sources to access the proximal femur allowing the reamer and even a long nail to be introduced.

The antegrade femoral nailing in our study was conducted by inserting the PFNA via the tip of the greater trochanter, an EP that is suggested to be advantageous (in terms of fluoroscopy times) over entering via the piriformis fossa [18]. Our choice of a suprapatellar EP for antegrade tibial nailing is consistent with current clinical practice, although this approach can cause cartilage and anterior cruciate ligament defects despite good fluoroscopic control [2]. The suprapatellar approach also results in greater EP accuracy compared to the parapatellar approach [19].

\section{Strengths and weaknesses}

One strength of this study is that we used the same sawbones with the same soft tissue envelope to compare times for different steps of the intramedullary nailing procedure. Four surgeons with varying levels of experience performed the procedures in random order and repeated all procedures three times for both image sources. These steps, however, are somewhat synthetic compared to the clinical setting. Furthermore, the need for imaging certain steps is potentially reduced in the not-so-obese sawbones or when there is no bleeding blurring the surgeon's view. We did not measure a full intramedullary nailing procedure; nor did we take the repositioning into account for total procedural time. The set up for a true lateral projection with the uniplanar device was not timed, which is a drawback for the comparison of times to DL. The number of swings was measured but only for the uniplanar C-arm.

\section{Conclusion}

The use of biplanar imaging slightly reduces the time to identify the proper EPs around the knee for retrograde femoral and antegrade tibial intramedullary nailing, although this time saving may not be clinically relevant. We also noted a concomitant reduction in radiation time to identify EPs in the tibia, but absolute differences in time were, again, small. We found no statistically significant or clinically relevant reduction in the time to identify the optimal EP in the greater trochanter or the time spent for DL during antegrade intramedullary nailing of the femur or tibia. Taken together, our findings suggest that the use of biplanar imaging may slightly reduce operating time and radiation exposure during defined steps of retrograde femoral and antegrade tibial intramedullary nailing, but that clinical benefits remain to be determined.

\section{Abbreviations}

AP: Anteroposterior; DL: Distal locking; EP: Entry point; PFNA: Proximal Femoral Nail Antirotation; SD: Standard deviations.

\section{Acknowledgements}

We thank Johan Ljungdahl, Jabbar Mohammed and Monika Olsson for help with performing the tests.

\section{Authors' contributions \\ PS, NPH and OW contributed to the study design. OW performed the statisti- cal analysis and wrote the first draft. PS, NPH and OW commented on and revised the manuscript. The final manuscript was read and approved by all authors.}

\section{Funding}

Open access funding provided by Uppsala University. Swemac Imaging has issued an unrestricted research grant. DePuy Synthes provided workshop instruments for intramedullary nailing. The funding bodies played no role in the design of the study and collection, analysis, and interpretation of data and in writing the manuscript.

\section{Availability of data and materials}

The datasets generated and/or analysed during the current study are available from the corresponding author on reasonable request.

\section{Declarations}

\section{Ethics approval and consent to participate}

This was a methodological phantom study without human subjects. The local radiation safety committee at Uppsala University Hospital was consulted. According to Swedish legislation no ethical application or radiation safety application was needed for this study according to the Ethics Review Act (2003:460) for research on human subjects or biological human samples. Consent not applicable.

\section{Consent for publication}

Not applicable.

\section{Competing interests}

OW received funding for this study from Swemac Imaging. PS and NPH declare no competing interests concerning this study.

Received: 24 June 2021 Accepted: 17 February 2022

Published online: 24 February 2022

\section{References}

1. Prasarn ML, Cattaneo MD, Achor T, Ahn J, Klinger CE, Helfet DL, Lorich DG. The effect of entry point on malalignment and iatrogenic fracture with the Synthes lateral entry femoral nail. J Orthop Trauma. 2010;24(4):224-9. https://doi.org/10.1097/BOT.0b013e3181 bedcc1.

2. Zamora R, Wright C, Short A, Seligson D. Comparison between suprapatellar and parapatellar approaches for intramedullary nailing of the tibia. Cadaveric study Injury. 2016;47(10):2087-90. https://doi.org/10.1016/j. injury.2016.07.024.

3. Westberry DE, Davids JR, Cross A, Tanner SL, Blackhurst DW. Simultaneous biplanar fluoroscopy for the surgical treatment of slipped capital femoral epiphysis. J Pediatr Orthop. 2008;28(1):43-8. https://doi.org/10.1097/BPO. Ob013e3181558bee.

4. Subramanian P, Goldie B. Biplanar imaging for intramedullary nailing of tibia and femur. Ann R Coll Surg Engl. 2013;95(6):447. https://doi.org/10. 1308/003588413X13629960048875m. 
5. Klug R, McCarthy JJ, Eilert RE. The use of a two C-arm technique in the treatment of slipped capital femoral epiphysis. Orthopedics. 2004;27(10):1041-2.

6. Granhed HP. A new technique of distal screw insertion for locked nailing. Acta Orthop Scand. 1998;69(3):320-1. https://doi.org/10.3109/17453 679809000939.

7. Pan S, Liu XH, Feng T, Kang HJ, Tian ZG, Lou CG. Influence of different great trochanteric entry points on the outcome of intertrochanteric fractures: a retrospective cohort study. BMC Musculoskelet Disord. 2017;18(1):107. https://doi.org/10.1186/s12891-017-1472-x.

8. Byun YS, Jung GH. Three-dimensional correlation between trochanteric fossa and the ideal entry point for antegrade femoral nailing. Injury. 2016;47(11):2539-43. https://doi.org/10.1016/j.injury.2016.09.026.

9. Oszwald M, Westphal R, Klepzig D, Khalafi A, Gaulke R, Muller CW, Wahl F, Krettek C, Gosling T. Robotized access to the medullary cavity for intramedullary nailing of the femur. Technol Health Care. 2010;18(3):17380. https://doi.org/10.3233/THC-2010-0580.

10. Crookshank MC, Edwards MR, Sellan M, Whyne CM, Schemitsch EH. Can fluoroscopy-based computer navigation improve entry point selection for intramedullary nailing of femur fractures? Clin Orthop Relat Res. 2014;472(9):2720-7. https://doi.org/10.1007/s11999-013-2878-X.

11. Yin $B$, Ahmad CS. Simultaneous use of $2 \mathrm{C}$-arm devices facilitates percutaneous treatment of proximal humerus fractures. Am J Orthop (Belle Mead NJ). 2012:41(10):477-9.

12. Schmucker A, Chen R, Vachhrajani S, Martinek M, Albert M. Radiation exposure in the treatment of pediatric supracondylar humerus fractures. Arch Orthop Trauma Surg. 2020;140(4):449-55. https://doi.org/10.1007/ s00402-019-03251-2.

13. Zuelzer DA, Allen J, Hsu JR, Matuszewski PE. The Far Side Opposite the Surgeon is Most Prone to Contamination From the C-Arm. J Orthop Trauma. 2019;33(12):e471-4. https://doi.org/10.1097/BOT.0000000000 001592.

14. Peters PG, Laughlin RT, Markert RJ, Nelles DB, Randall KL, Prayson MJ. Timing of C-arm drape contamination. Surg Infect (Larchmt). 2012;13(2):1103. https://doi.org/10.1089/sur.2011.054.

15. Momenzadeh K, Williams C, Czerwonka N, Kwon JY, Nazarian A, Miller CP. Contamination of the Mini C-Arm During Foot and Ankle Surgery. Foot Ankle Int. 2021;42(8):994-1001. https://doi.org/10.1177/1071100721 1001032.

16. Gershkovich GE, Tiedeken NC, Hampton D, Budacki R, Samuel SP, Saing M. A Comparison of Three C-Arm Draping Techniques to Minimize Contamination of the Surgical Field. J Orthop Trauma. 2016;30(10):e351-6. https:// doi.org/10.1097/BOT.0000000000000619.

17. Darbandi A, Pujari A, Lin C. Evaluation of a novel C-Arm draping technique to minimize surgical field contamination and surgery time. ANZ J Surg. 2021. https://doi.org/10.1111/ans.17013.

18. Sheth U, Gohal C, Chahal J, Nauth A, Dwyer T. Comparing Entry Points for Antegrade Nailing of Femoral Shaft Fractures. Orthopedics. 2016;39(1):e43-50. https://doi.org/10.3928/01477447-20151218-09.

19. Anderson TRE, Beak PA, Trompeter AJ. Intra-medullary nail insertion accuracy: A comparison of the infra-patellar and supra-patellar approach. Injury. 2019;50(2):484-8. https://doi.org/10.1016/j.injury.2018.12.024.

\section{Publisher's Note}

Springer Nature remains neutral with regard to jurisdictional claims in published maps and institutional affiliations.

Ready to submit your research? Choose BMC and benefit from:

- fast, convenient online submission

- thorough peer review by experienced researchers in your field

- rapid publication on acceptance

- support for research data, including large and complex data types

- gold Open Access which fosters wider collaboration and increased citations

- maximum visibility for your research: over $100 \mathrm{M}$ website views per year

At $\mathrm{BMC}$, research is always in progress.

Learn more biomedcentral.com/submissions 